
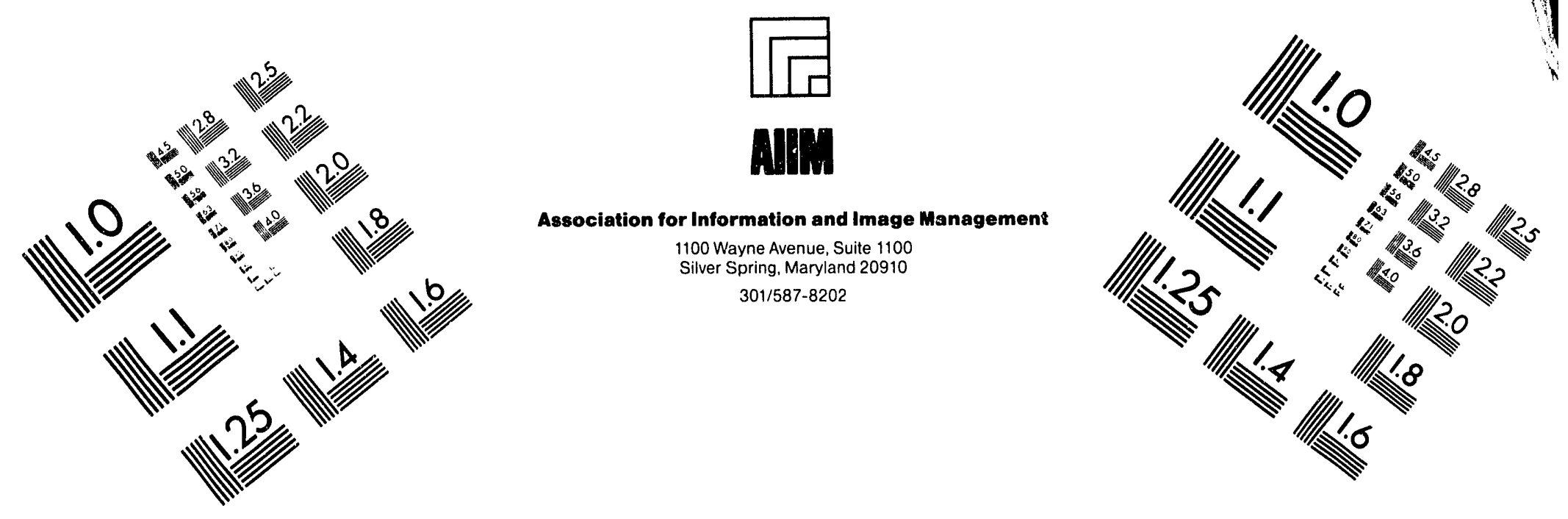

\title{
Centimeter
}

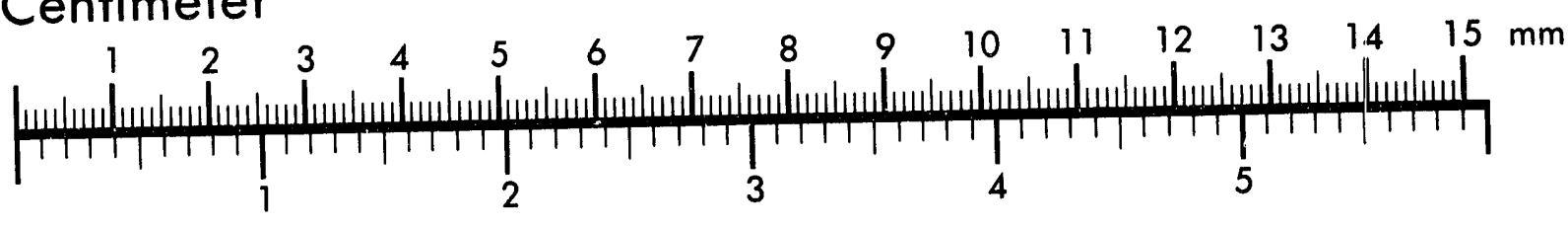
Inches
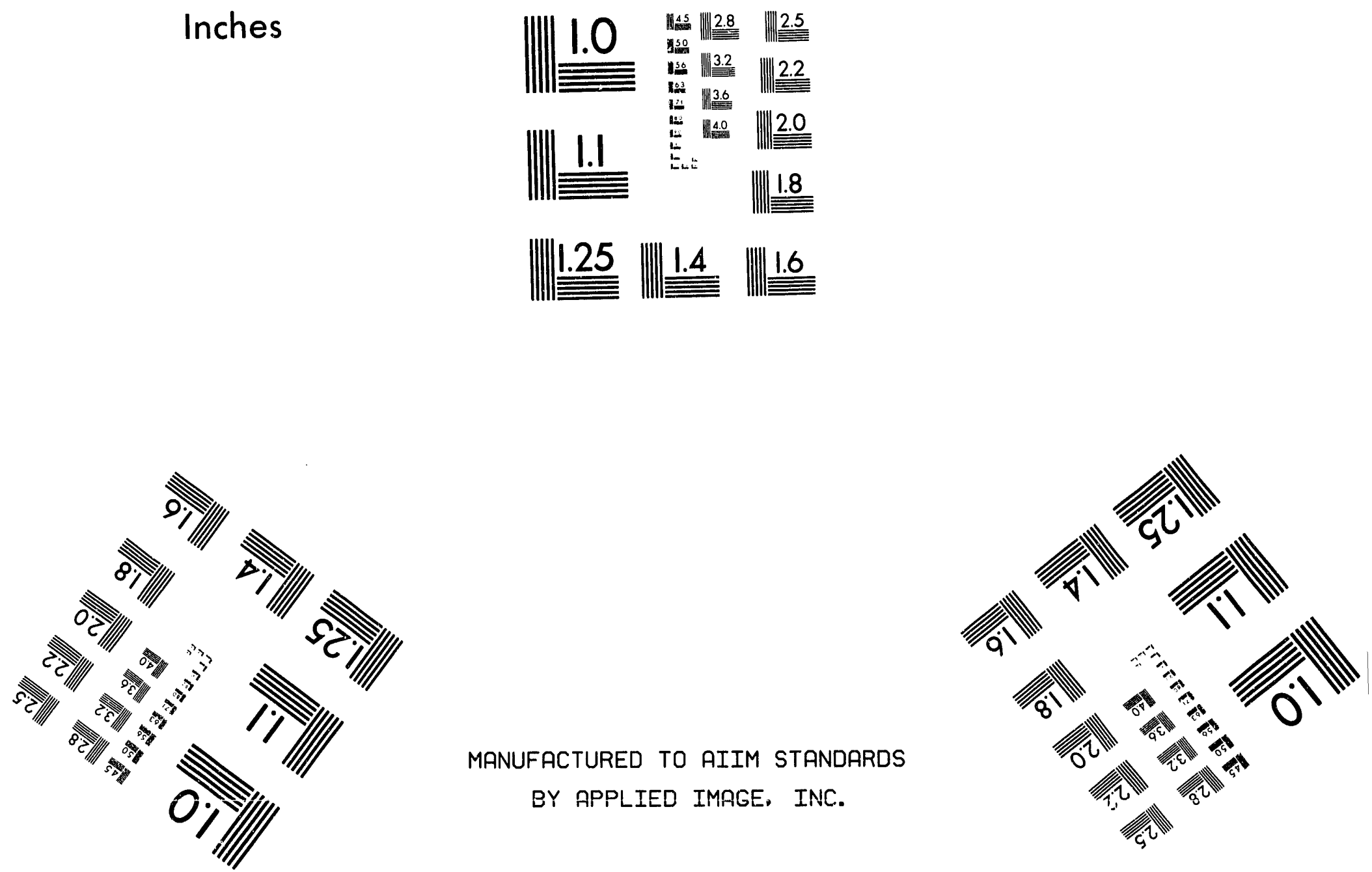

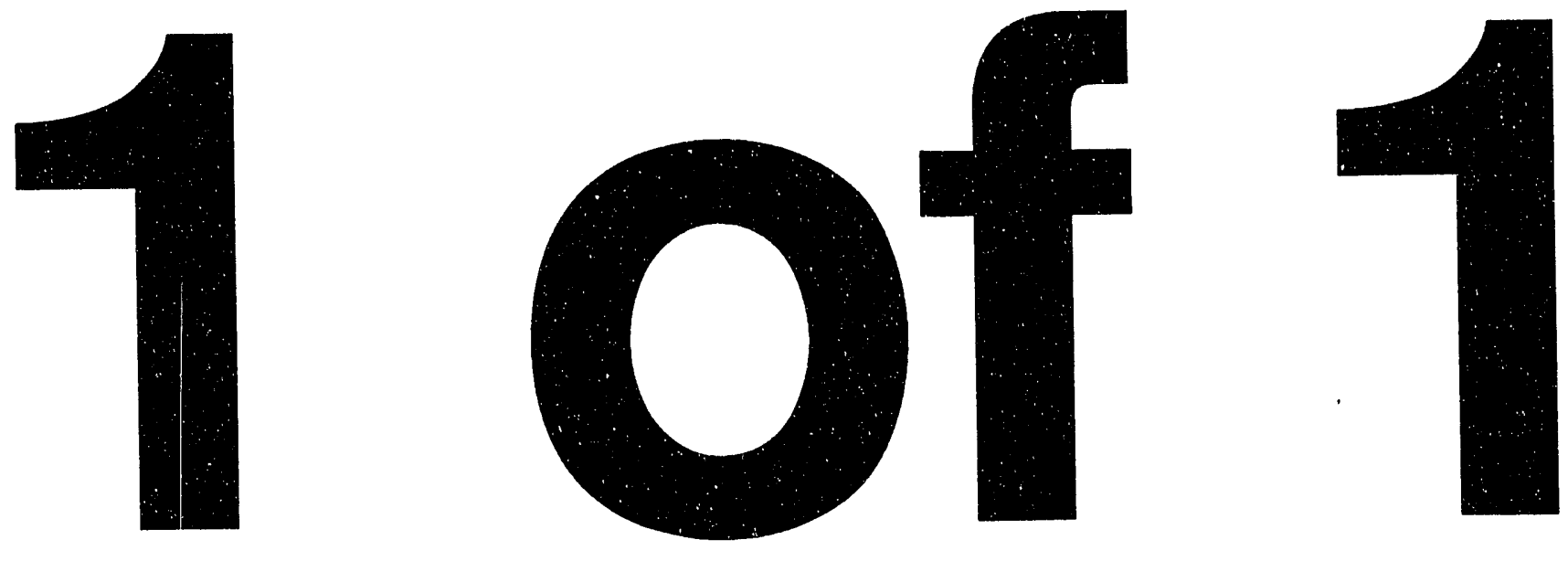
UCRL-JC-110875

PREPRINT

\section{Design of a Formaldehyde Photodissociation Process for Carbon and Oxygen Isotope Separation}

Richard C. Stern

Karl F. Scheibner

This paper was prepared for submittal to

Laser Isotope Separation,

SPIE's International Symposium on Lasers, Sensors, and Applications

Los Angeles, California

January 16-23, 1993

January 20, 1993

This is a preprint of a paper intended for publication in a joumal or proceedinga. Since changes may be made before publication, this preprint is made available with the understanding that it will not be cited or reproduced without the permission of the author.

\section{,}

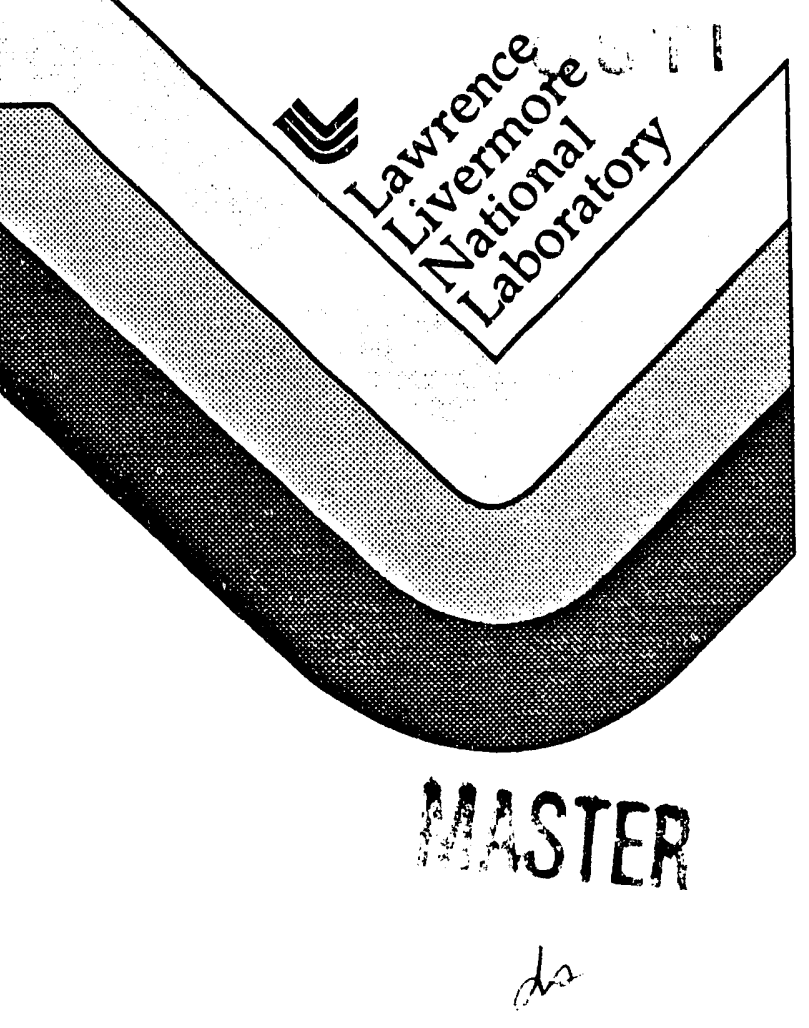




\section{DISCLAMMER}

This document was prepared as an account of work sponsored by an agency of the United States Government. Nelther the United States Government nor the Univeraity of California nor any of their employees, makes any warranty, express or implied, or assumes any legal liability or responsibility for the accuracy, completeness, or usefuliness of an y information, apparatus, produch or process disclosed, or represents that its use would not infringe privately owned rights. Reference hereln to any specific commercial products, process, or service by trade name, trademark, manufacturer, or otherwise, does not necessarily constitute or imply its endorsement, recommendation, or favoring by the United States Government or the University of California. The views and opinions of authors expressed herein do not necessarily state or reflect those of the United States Government or the University of California, and shall not be used for advertising or product endorsement purposes. 
Design of a formaldehyde photodissociation process for carbon and oxygen isotope separation

\author{
Richard C. Stern \& Karl F. Scheibner \\ Lawrence Livermore National Laboratory \\ 7000 East Avenue \\ Livermore, CA 94550-9900
}

\begin{abstract}
The current shortage of ${ }^{18} \mathrm{O}$ has revived interest in using one step UV photodissociation of formaldehyde to enrich ${ }^{13} \mathrm{C},{ }^{17} \mathrm{O}$ and ${ }^{18} \mathrm{O}$. The frequency doubled output of the copper laser pumped dye laser system currently in operation at LINL can be used to drive this dissociation. We use a simple kinetics model and our experience with Atomic Vapor Laser Isotope Separation (AVLIS) process design to examine the relative merits of different designs for a formaldehyde photodissociation process. Given values for the molecular photoabsorption cross section, partition function, spectroscopic selectivity, collisional exchange and quenching cross sections (all as parameters), we perform a partial optimization in the space of illuminated area, formaldehyde pressure in each stage, and formaldehyde residence time in each stage. We examine the effect of cascade design (heads and tails staging) on molecule and photon utilization for each of the three isotope separation missions, and look in one case at the system's response to different ratios of laser to formaldehyde costs. Finally, we examine the relative cost of enrichment as a function of isotope and product assay. Emphasis will be as much on the process design methodology, which is general, as on the specific application to formaldehyde.
\end{abstract}

\title{
1. DESIGN PARAMETERS
}

The design of a single stage of a laser isotope enrichment process involves the choice of an operating point for the stage. In the restricted context considered here, the total laser power, laser PRF, photolysis cell length, and the number of laser passes through the cell will be taken as fixed, having been set by the available hardware and a single global optimization. The process designer then selects the area illuminated by a single pass (laser footprint), the formaldehyde pressure in each stage (temperature is fixed), and the formaldehyde flow velocity down the cell in each stage (residence time). The tradeoffs involved in setting these parameters are familiar: a larger area leads to lower fluence, hence lower saturation and hetter photoselectivity, at the cost of lower molecular conversion per pulse; higher pressure increases laser absorption and photon utilization, at the cost of increased excitation exchange; lower flow velocity increases atom utilization, at the cost of reduced product quantity and assay due to bleaching.

Using the parameters we expect for UV photodissociation of formaldehyde, a single stage of enrichment is capable of producing $\sim 70 \%{ }^{13} \mathrm{C}$ from $1.11 \%$ feed, or $-40 \%{ }^{18} \mathrm{O}$ from $0.208 \%$ feed, or $\sim 7 \% 170$ from $0.038 \%$ feed. The demand spectrum for these isotopes calls for higher enrichments. To reach $95 \%$ product assay requires 2 stages for ${ }^{13} \mathrm{C}$ and ${ }^{18} \mathrm{O}, 3$ stages for 170. The variables in a multi-stage cascade can be displayed in a pair of tables, one for the product wiring, and one for the tails wiring. The product table for the simple case of one feed stage (stage 0 ), one product stage (stage +1 ), and no tails stages, looks like

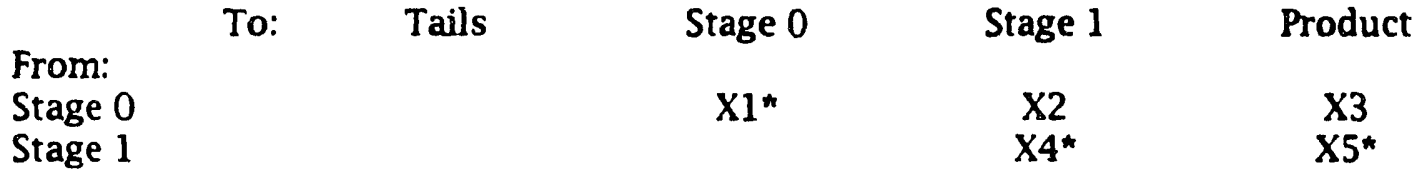


and the corresponding tails wiring looks like

$\begin{array}{lccccc} & \text { To: } & \text { Tails } & \text { Stage } 0 & \text { Stage } 1 & \text { Product } \\ \text { From: } & & \mathrm{Y}^{\star} & \mathrm{Y}^{\star} & & \\ \text { Stage 0 } & & \mathrm{Y}^{\star} & \mathrm{Y}^{\star} & \mathrm{Y}^{\star} & \end{array}$

The entries in each row give the fraction of the product (first table) or tails (second table) from the stage in the left hand column which goes as feed to the stage indicated in the column header, or which exits the system as cascade (vs. stage) product or tails. Each row is normalized to 1 , so there are 3 free variables in the product wiring, and 3 free variables in the tails wiring of a 2 stage cascade.

For the low feed cost and high stage enrichment factors ( $\left.\beta^{\prime} s\right)$ found in formaldehyde photodissociation, we can fix 5 of these 6 degrees of freedom a priori. The optimum tails cascade sends all tails as feed to the stage below (or all tails out of the cascade from the feed stage), since the upper stage tails are too enriched to be discarded, while feed is too inexpensive for refluxing tails back into the stage that produced them. Likewise, as long as the number of product stages is adequate, it is always preferable to increase product assay by using another product stage, rather than by refluxing product to feed in the same stage. These arguments fix all the variables marked with asterisks in the tables above at either 0 or 1 . The remaining degree of freedom is the fraction of the stage 0 product that is fed to stage 1 . It is worth noting that if this fraction becomes 0 , stage 1 has no feed, and the cascade drops to a single stage. This ability to drop stages during the optimization process is a general feature of the method.

We have defined an optimization problem that has 6 free design parameters for a 2 stage cascade (1 cascade wiring parameter, 1 laser area, 2 stage pressures and 2 stage flow velocities). By similar arguments, a 1 stage cascade has 3 free variables, and a 3 stage cascade, the most complicated case considered here, has 10 degrees of freedorn.

\section{OPTIMIZATION METHOD}

The objective function optimized is the annual profit of the enrichment enterprise. Annual costs are the sum of laser fixed costs, separator fixed costs, separator costs which scale with the maximum molar flow rate through any stage (thereby scaling the required capacity of the flow system), and formaldehyde costs which scale with the amount of formaldehyde used as either feed or blend stock. Capital equipment is amortized. Laser costs are based on the well documented AVLIS copper laser and dye laser chains, along with a projected doubling efficiency and UV transport loss. These costs are, of course, for photons delivered to the separator. The photon utilization in the separator is a dependent parameter in the optimization. Feed costs are based on formaldehyde purchase and recovery as formalin. We assume tails can be returned for a credit of $90 \%$ of the purchase price. The amount of formaldehyde which is actually consumed (converted into product) is a negligible fraction of the feed. For one typical case (70\% 180 product), costs divide as: lasers $67.0 \%$, separator fixed, $27.4 \%$, separator flow, $3.0 \%$, and feed used, $2.6 \%$.

Sales are made against a specified demand for a given number of moles of enriched isotope at a given assay and price per mole. For these studies, only one assay is requested, at a quantity larger than any possible output of the enrichment facility. The demand assay is met exactly by blending the raw cascade outputs. The output stream with assay above and closest to the demand is blended with the output stream with assay below and closest (or failing such 
a stream, with natural feed). If there is no cascade output at an assay above the demand, no sales are made. Note that the raw cascade "product" and "tails" outputs can have different fates after blending, since a cascade tails stream at an assay above natural abundance can be blended into plant product and a cascade product stream which finds no market is a fortiori part of the plant tails.

Optimization is performed by simulated annealing. 1 The annealing temperature and the size of the quantum of change in the parameters are decreased following schedules which have been tuned to give reasonable approximations to the optimum for a reasonable expenditure of computer time. Each case requires 2000 to 5000 plant evaluations, and is repeated 5 times to sample the variation in the final result. In general, we find several different parameter sets which give nearly the same annual profit. The cascade wiring tends to be tightly determined and the pressures relatively tightly determined, while the illuminated area and the flow velocities can trade against each other over wide ranges (making them both less interesting as reported results, and less critical for the plant design).

\section{FORMALDEHYDE PHOTODISSOCIATION}

Single photon absorption by formaldehyde in the $290-355 \mathrm{~nm}$ wavelength region can lead to isotopically selective dissociation into stable molecular products, $\mathrm{H}_{2}$ and $\mathrm{CO}$, as discovered and characterized by Moore and coworkers. ${ }^{2}$ On the basis of this work, we have chosen the molecular parameters in the following table, with the recognition that absorption cross sections and dissociation lifetimes can vary sharply from one excited state to the next, and could be quite different for a particular transition of interest. The photoabsorption cross section is for molecules in the lower state of the transition. The excitation xxchange cross section is derived from our reanalysis of early experiments on $C$ and $O$ enrichment. ${ }^{3}$ Quenching is subsumed into a universal quantum yield, and is not treated as a collisional process. Zero-fluence spectroscopic selectivities up to 450 have been measured in ${ }^{14} \mathrm{C}^{4}$ We assume a spectroscopic selectivity of 300 for ${ }^{18} \mathrm{O}$ ( 2 amu difference), and 200 for ${ }^{13} \mathrm{C}$ and ${ }^{17} \mathrm{O}$ (1 amu difference).

\begin{tabular}{|l|l|}
\hline Formaldehyde Photodissociation Parameters & \\
\hline Photoabsorption Cross Section & \\
\hline Spectroscopic Selectivity & $5.0 \times 10^{-17} \mathrm{~cm}^{2}$ \\
\hline Excitation Exchange Cross Section & $200(13 \mathrm{C}, 17 \mathrm{O}), 300(18 \mathrm{O})$ \\
\hline S1 to SO* Crossing Rate & $2.8 \times 10^{-16} \mathrm{~cm}^{2}$ \\
\hline Fraction Available & $2.5 \times 10^{6} \mathrm{~s}^{-1}$ \\
\hline Quantum Yield & $5 \times 10^{-3}$ \\
\hline
\end{tabular}

The simple photodissociation kinetics model built around these parameters is illustrated in the following figure. While the laser pulse is on $(40 \mathrm{~ns})$, only the independent optical pumping of the isotopic and non-isotopic molecules is considered. After the pulse, the $S_{1}$ populations decay by excitation exchange with the opposite ground state, and by spontaneous crossing into $S_{0}{ }^{*}$, followed immediately by dissociation. The kinetics model is solved anew, following the path of the laser as it folds through the vapor, each time the number of photons in the laser pulse or the number of undissociated isotopic molecules changes by more that a preset tolerance. 
Kinetics During Laser Pulse

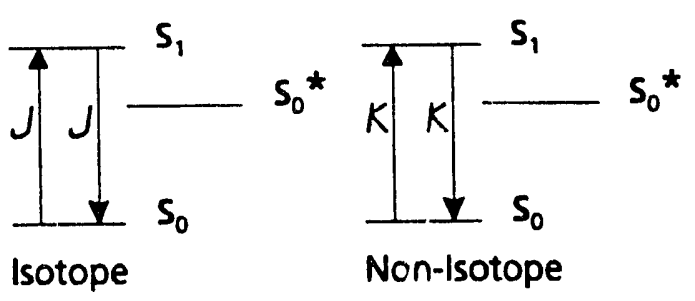

Kinetics After Laser Pulse

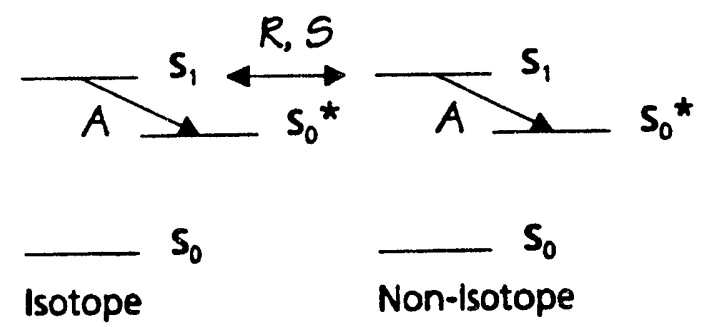

The kinetics model is deliberately kept simple, since, in the course of finding one optimum design, it typically will be solved $\sim 1.6 \times 10^{8}$ times (200 steps per cell length $\times 10$ laser passes $\times 2$ stages per cascade $\times 2$ sweeps to converge the cascade solution $\times 4000$ solutions per simulated annealing $x 5$ replications of each case)! The most significant approximation is the neglect of excitation exchange during the laser pulse. However, by the time this effect becomes important, excitation exchange after the laser pulse will have severely degraded performance and the optimization will avoid these regimes.

\section{STAGING AND OPERATING POINTS}

All the cases presented here are solved using the following common parameters:

\begin{tabular}{|l|l|}
\hline Common Design Parameters & \\
\hline & \\
\hline Laser Power & $129 \mathrm{~W}$ \\
\hline Laser PRF & $4350 \mathrm{~Hz}$ \\
\hline Laser Pulse Length & $40 \mathrm{~ns}$ \\
\hline Temperature & $300 \mathrm{~K}$ \\
\hline Photolysis Cell Length & $10 \mathrm{~m}$ \\
\hline Number of Laser Passes & 10 \\
\hline Mirror Reflectivity & 0.99 \\
\hline System Availability & $90 \%$ \\
\hline
\end{tabular}

The laser power is the frequency doubled dye-laser-chain power delivered to the separator from a system pumped by one AVLIS copper laser oscillator and one AVLIS copper laser amplifier. The laser PRF and pulse length are determined by the copper lasers. The number of laser passes is a significant design parameter which is not optimized in this study. The 10 laser passes fold through 6 parallel vapor streams. The first and last vapor streams are illuminated once per laser pulse (exactly in the model, on the average in the real geometry), the central four passes are illuminated twice. Different stages time share the same apparatus, each using a fraction of the available time proportional to its feed quantity (as determined by the cascade wiring) divided by its feed rate (as determined by the laser area, the number of parallel vapor streams, the stage pressure and the stage flow velocity).

The most basic results of the study are the staging, the number and assay of cascade product streams, and the quantity of blended product for each isotope at a variety of demand 
assays. All this for a plant of fixed "size" as defined by the laser power and cell length. These results are shown in the following composite figure.

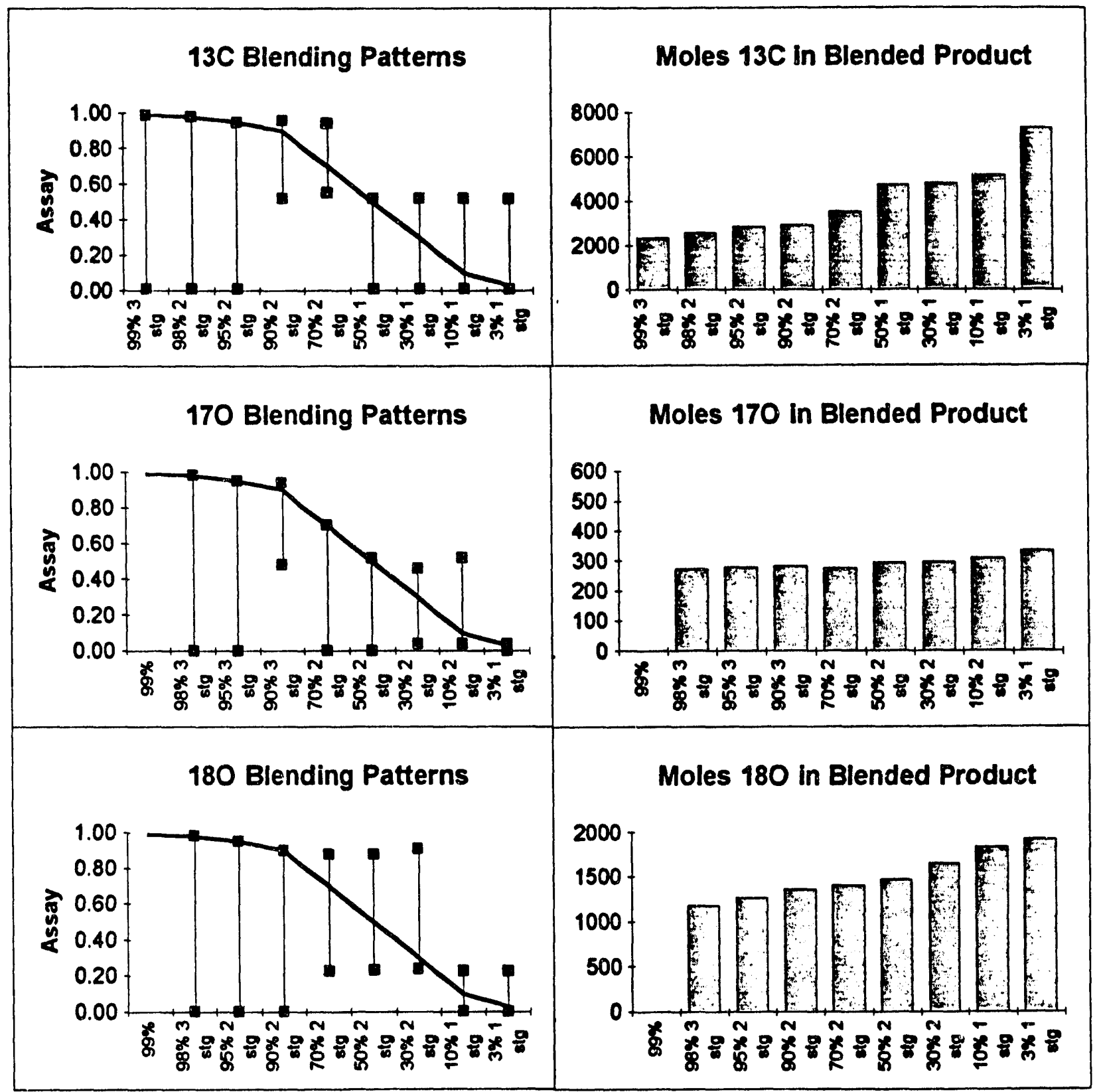

Calculations are performed for blended product assays of $99 \%\left({ }^{13} \mathrm{C}\right.$ only), $98 \%, 95 \%$, $90 \%, 70 \%, 50 \%, 30 \%, 10 \%$ and $3 \%$. The abscissa of each plot reports these assays, and the number of stages in the optimized cascade. The left hand figure in each row reports the blending pattern. The solid line in each of these figures repeats the target demand assay from the abscissa. The boxes are the cascade output assays. The tie lines connect the two raw assays that are blended to match the demand assay. For example, in $180,98 \%, 95 \%$ and $90 \%$ assays are met by blending the sole cascade product stream with a negligible amount of feed, the raw cascade is essentially putting out the required assay. For $70 \%, 50 \%$ and $30 \%$ demanded, the cascade blends second stage and first stage product, largely second stage at $70 \%$ demand assay, almost entirely first stage at $30 \%$. The cascade wiring changes accordingly. In all cases, 
for all isotopes, we find essentially no wasted product. All the raw cascade product is consumed in the blends.

One interesting optimization which can be read from these figures is the trade between single stage assay and throughput. In the limit of zero fluence and zero pressure (no saturation or excitation exchange), the product and feed assays for a stage are related by

$$
X_{P}=\frac{X_{F}}{X_{F}+\frac{\left(1-X_{F}\right)}{S}}
$$

where $S$ is the spectroscopic selectivity. The following table compares these zero-throughput assays with the optimum assays found at finite throughputs.

\begin{tabular}{|l|l|l|}
\hline Stage 0 Product Assays & & \\
\hline & & \\
\hline Isotope & Limiting Assay & Optimum Assay \\
\hline $13 \mathrm{C}$ & $69.2 \%$ & $52-55 \%$ \\
\hline $17 \mathrm{O}$ & $7.1 \%$ & $4 \%$ \\
\hline $18 \mathrm{O}$ & $38.5 \%$ & $22-24 \%$ \\
\hline
\end{tabular}

The right hand figure in each row above gives the amount of the enriched isotope in the blended product. At low blended assays, the product quantities are approximately in the ratios of the natural abundances of each isotope, as one would naively expect. At high assays, the ${ }^{18} \mathrm{O}$ and particularly the ${ }^{13} \mathrm{C}$ product quantities fall below the natural abundance ratio. This shortfall will be seen in other guises below. As to its cause, our present speculation is that the global values for the photolysis cell length and the number of passes, which we imposed above, may be way off optimum for some stages. Systems processing 170 at $0.038 \%$ feed assay and ${ }^{13} \mathrm{C}$ at $52 \%$ feed assay may benefit from different laser path lengths.

\section{ATOM AND PHOTON UTILIZATIONS}

The next set of figures show the utilization of isotopic atoms and of photons from the optimum cases, as a function of demand product assay, for each of the isotopes.

The results are qualitatively as expected. With the cost schedules we are using, a mole of photons costs 240 times as much as a mole of ${ }^{13} \mathrm{C}$ containing formaldehyde, 8 times as much as a mole of 170 containing formaldehyde, and 45 times as much as a mole of 180 containing formaldehyde. Therefore, stage 0 atom utilizations should be low and stage 0 photon utilizations should be high, as indeed they are. The atoms fed to stages 1 and 2 have the cost of one or more photons incorporated into them, and therefore should be utilized much more efficiently, as they are. Qualitatively, there are some puzzles. The optimum stage 0 atom utilizations are all roughly the same $(4 \%-8 \%)$, where the cost ratios above would suggest a larger spread. Stage 2 atom utilizations appear low (although the atoms not utilized here return to stage 1). 


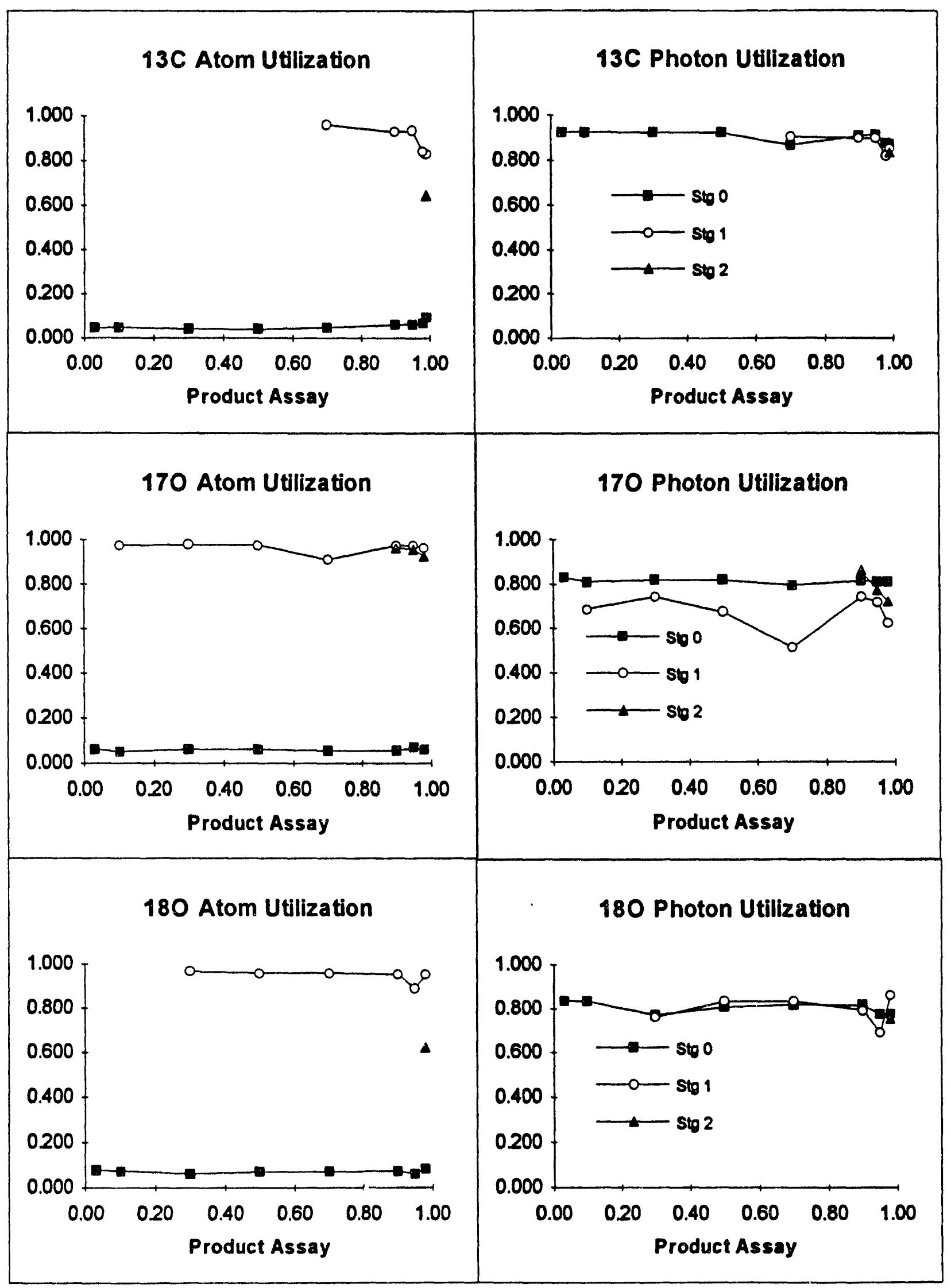




\section{FEED COSTS AND TAILS ASSAYS}

As an aside, we have examined the effects of altering the ratio of photon to isotope costs by taking one typical case, 180 at $70 \%$ demand assay, and increasing the feed costs by ratios of $1 x$ (reference), $5 x, 10 x, 20 x$, and $30 x$. The illuminated area per pass is held constant for this series, near the optimum value previously found for the $1 x$ case. The optimum stage 0 pressure and photon utilization remain remarkably constant through the series (at 3.7 - 3.75 Torr and $81 \% \cdot 82 \%$ respectively). Major variations are shown in the table.

\begin{tabular}{|l|l|l|l|}
\hline $\begin{array}{l}\text { Effect of Feed Cost } \\
\text { Variation }\end{array}$ & $180 \cdot 70 \%$ Product & & \\
\hline $\begin{array}{l}\text { Photon Cost } / 18 \mathrm{O} \\
\text { Cost }\end{array}$ & Tails Assay & $\begin{array}{l}\text { Atom } \\
\text { Utilization }\end{array}$ & $\begin{array}{l}\text { Flow Velocity }- \text { Stg } \\
0 \mathrm{~cm} / \mathrm{s}\end{array}$ \\
\hline 44.9 & $0.197 \%$ & $5.3 \%$ & 10800 \\
\hline 9.0 & $0.186 \%$ & $10.9 \%$ & 5200 \\
\hline 4.5 & $0.175 \%$ & $15.9 \%$ & 3400 \\
\hline 2.2 & $0.166 \%$ & $20.3 \%$ & 2600 \\
\hline 1.5 & $0.158 \%$ & $24.5 \%$ & 2100 \\
\hline
\end{tabular}

Atom utilization rises and the tails assay drops as feed becomes relatively more expensive. The model does this by dropping the stage 0 flow velocity and increasing the number of laser pulses each formaldehyde molecule sees. In each of the cases in this study, a tails stripping stage below the feed stage (stage -1) was made available. In every case, the optimization set the feed to the tails stage to 0 , i.e., even at the highest price examined here, formaldehyde is not valuable enough to warrant a special stage to save it.

\section{PRODUCT COSTS}

The last set of results we will examine are the ones of the greatest practical interest. How do the enterprise's costs of generating product vary with product assay, or from isotope to isotope? These answers presumably determine the enterprise's price schedules.

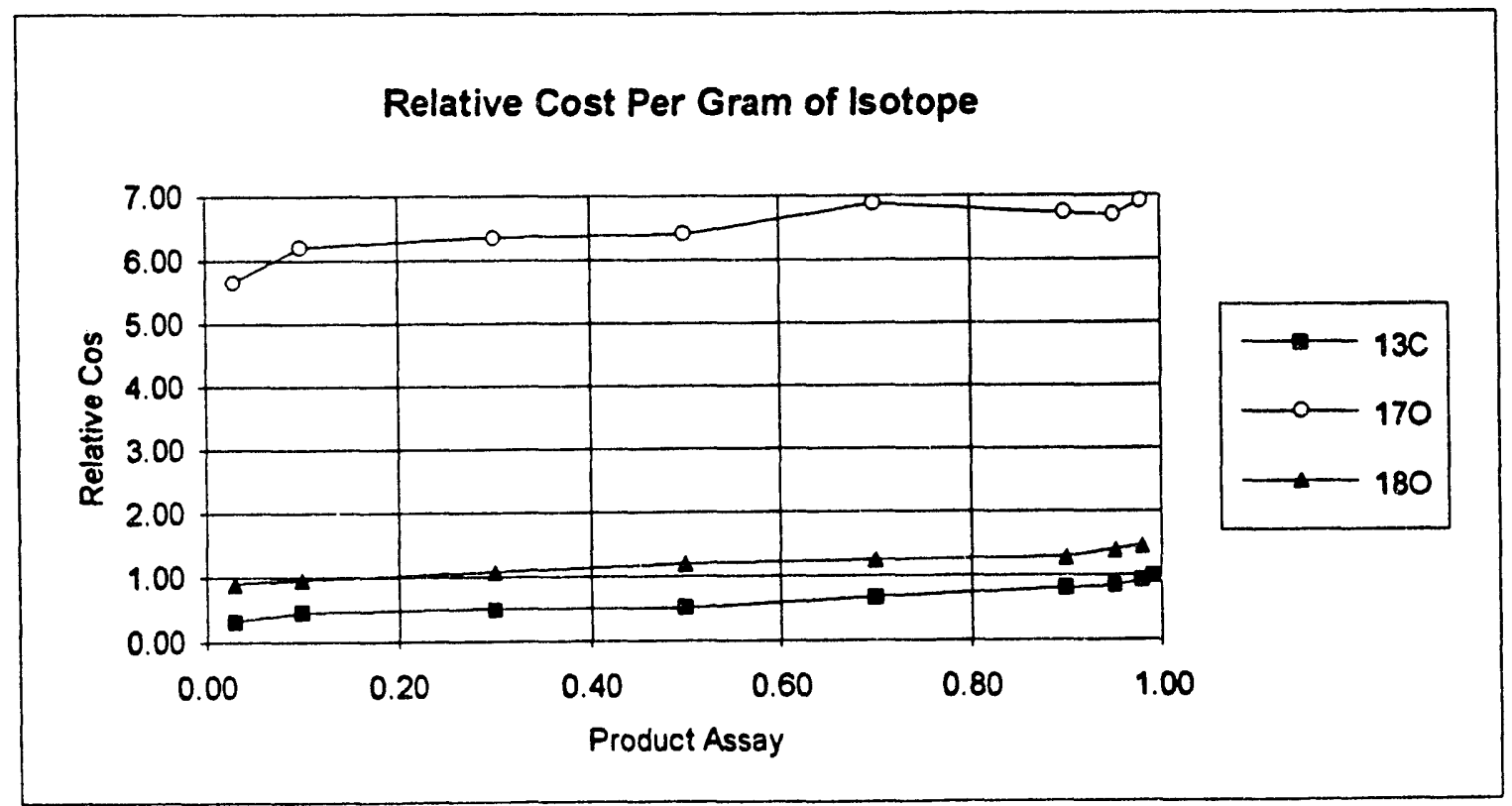


The figure above shows the costs in the units usually seen for product prices, $\mathbf{S / g}$ of isotope. The pattern is qualitatively as expected. High assays cost more per gram of isotope than low assays (otherwise one would produce all lower assays by blending the highest obtainable assay). ${ }^{13} \mathrm{C}$ is the least expensive, ${ }^{17} \mathrm{O}$ is the most expensive. To see if these costs are "right", we redisplay them on a per mole basis, and normalize out the different natural abundances.

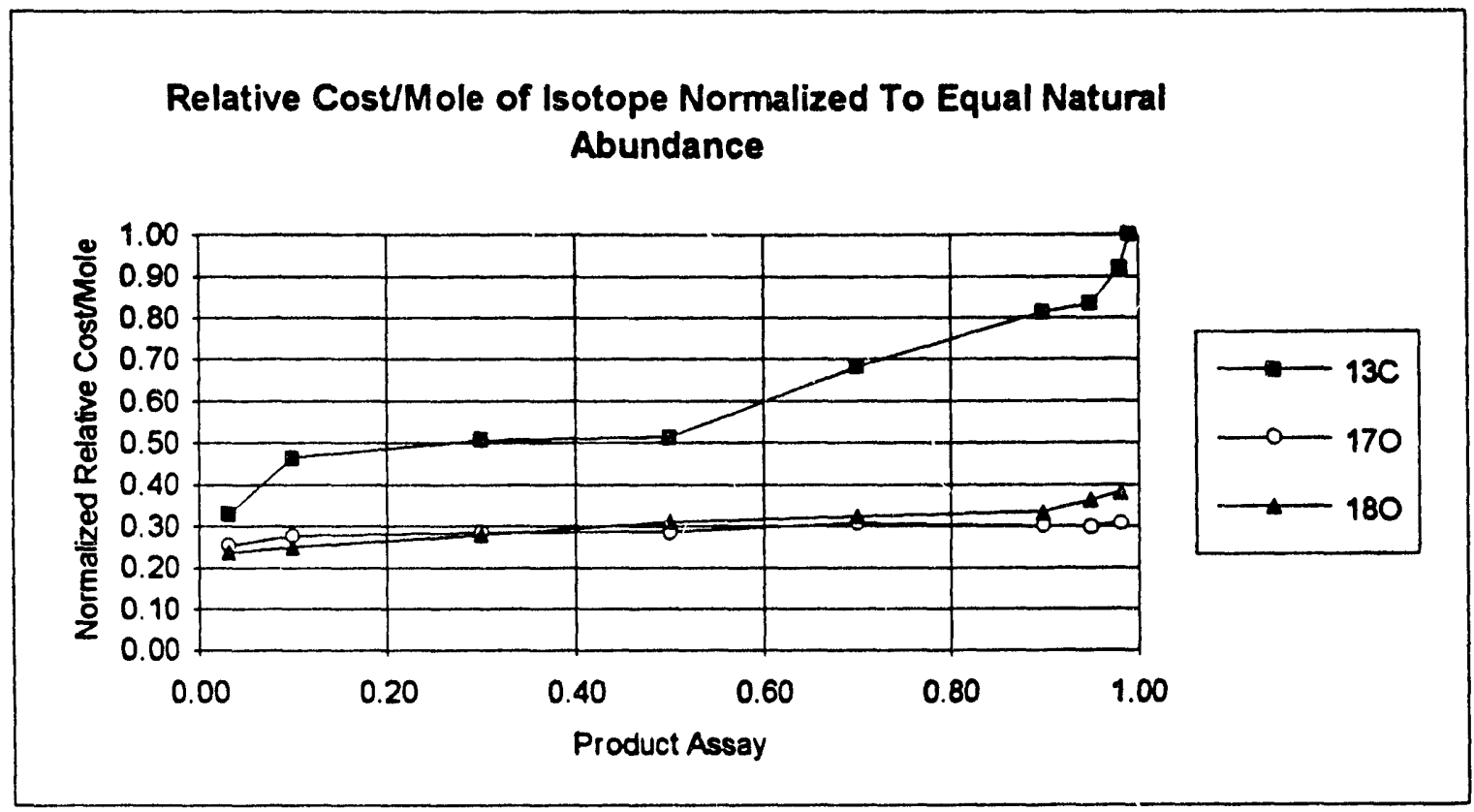

As displayed in this fashion, ${ }^{13} \mathrm{C}$ costs appear to be relatively too high, particularly at high assays. ${ }^{18} \mathrm{O}$ costs are also probably higher than they should be, given the higher ${ }^{18} \mathrm{O}$ spectroscopic selectivity. This discussion assumes that the normalized product costs should, in fact, be the same for different isotopes, a question we plan to pursue.

\section{ACKNOWLEDGMENT}

Work performed under the auspices of the U.S. Department of Energy by Lawrence Livermore National Laboratory under contract number W-7405-ENG-48.

\section{REFERENCES}

1. W.H. Press, B.P. Flannery, S.A. Teukolsky and W.T. Vetterling, Numerical Recipes, Chapter 10.9, Cambridge University Press, Cambridge, 1986.

2. C.B. Moore and J. C. Weisshaar, "Formaldehyde Photochemistry", Ann. Rev. Phys. Chem. 34, 525-555, 1983.

3. J. Marling, "Isotope separation of oxygen-17, oxygen-18, carbon-13, and deuterium by ion laser induced formaldehyde photopredissociation, J. Chem. Phys. 66, 4200-4225, 1977.

4. R.E.M. Hedges, P. Ho and C.B. Moore, "Enrichment of Carbon-14 by Selective Laser Photolysis of Formaldehyde, Appl. Phys. 23, 25-32, 1980. 

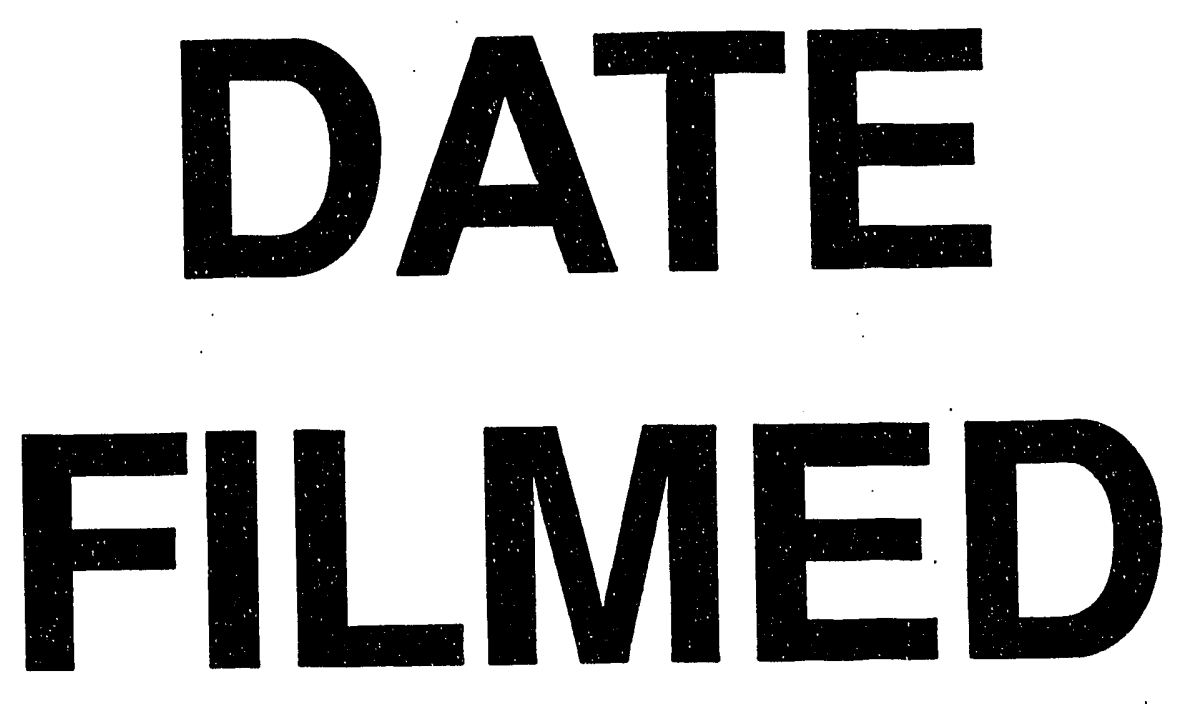

$8 / 17 / 93$
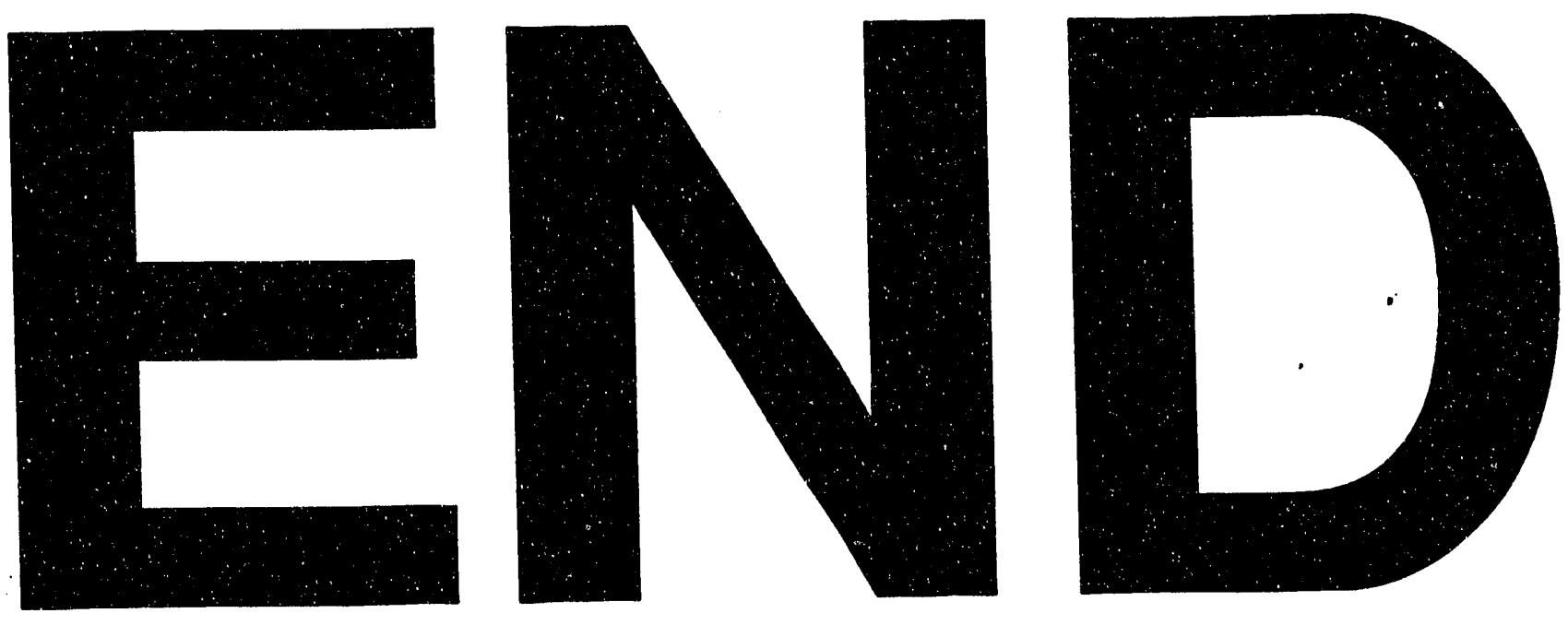
